\title{
RESPIRATORY DYSFUNCTION IN UNSEDATED DOGS WITH GOLDEN RETRIEVER MUSCULAR DYSTROPHY
}

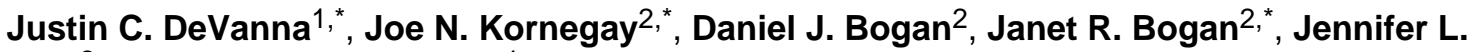 \\ Dow $^{2}$, and Eleanor C. Hawkins ${ }^{1}$ \\ ${ }^{1}$ Department of Clinical Sciences, College of Veterinary Medicine, North Carolina State \\ University, Raleigh, NC, 27607, United States. \\ ${ }^{2}$ Departments of Pathology and Laboratory Medicine and Neurology and the Gene Therapy \\ Center, School of Medicine, University of North Carolina, Chapel Hill, NC, 27599, United States.
}

\begin{abstract}
Golden retriever muscular dystrophy (GRMD) is a well-established model of Duchenne muscular dystrophy. The value of this model would be greatly enhanced with practical tools to monitor progression of respiratory dysfunction during treatment trials. Arterial blood gas analysis, tidal breathing spirometry, and respiratory inductance plethysmography (RIP) were performed to determine if quantifiable abnormalities could be identified in unsedated, untrained, GRMD dogs. Results from 11 dogs with a mild phenotype of GRMD and 11 age-matched carriers were compared. Arterial blood gas analysis was successfully performed in all dogs, spirometry in 21 of $22(95 \%)$ dogs, and RIP in 18 of 20 (90\%) dogs. Partial pressure of carbon dioxide and bicarbonate concentration were higher in GRMD dogs. Tidal breathing peak expiratory flows were markedly higher in GRMD dogs. Abnormal abdominal motion was present in 7 of 10 (70\%) GRMD dogs. Each technique provided objective, quantifiable measures that will be useful for monitoring respiratory function in GRMD dogs during clinical trials while avoiding the influence of sedation on results. Increased expiratory flows and the pattern of abdominal breathing are novel findings, not reported in people with Duchenne muscular dystrophy, and might be a consequence of hyperinflation.
\end{abstract}

\section{Keywords}

Muscular dystrophy; golden retriever muscular dystrophy; pulmonary function test; respiratory inductance plethysmography; spirometry; blood gas analysis; flow-volume loop

(C) 2013 The Authors. Published by Elsevier B.V. All rights reserved.

Corresponding author: Dr. Eleanor C. Hawkins, Department of Clinical Sciences, North Carolina State University, 1060 William Moore Drive, Raleigh, NC, 27607, United States Tel.: 919-513-7727; fax: 919-513-6336. eleanor_hawkins@ncsu.edu.

${ }^{*}$ Changes of address: Justin C. DeVanna, DVM, MSpVM; Public Health Command Region-South, 2899 Schofield Rd, Joint Base San Antonio Fort Sam Houston, TX 78234, United States.

Joe N. Kornegay, DVM, PhD; Department of Veterinary Integrative Biosciences, (Mail Stop 4458), College of Veterinary Medicine, Texas A\&M University, College Station, TX, 77843-4458, United States.

Janet R. Bogan,BS; Department of Clinical Sciences, College of Veterinary Medicine, North Carolina State University, Raleigh, NC 27607, United States.

5. Conflict of interest The authors declare that they have no conflict of interest.

Publisher's Disclaimer: This is a PDF file of an unedited manuscript that has been accepted for publication. As a service to our customers we are providing this early version of the manuscript. The manuscript will undergo copyediting, typesetting, and review of the resulting proof before it is published in its final citable form. Please note that during the production process errors may be discovered which could affect the content, and all legal disclaimers that apply to the journal pertain. 


\section{Introduction}

Duchenne muscular dystrophy (DMD) is the most common muscular dystrophy of people, with incidence per 100,000 male live births ranging from 13.0 - 28.2.[1,2] It is an X-linked, recessive disease caused by a mutation in the gene encoding dystrophin. The disease is ultimately fatal, with respiratory failure accounting for up to $80 \%$ of deaths.[3,4] Respiratory failure results from progressive ventilatory compromise, often complicated by bronchial infection or pneumonia. [5] Breathing is impaired by weakness of the respiratory muscles and decreased chest wall and lung compliance.[4-7] Sleep-disordered breathing, upper airway obstruction, and other factors might contribute to nocturnal hypoventilation.[4,8,9] Macroglossia, reported in one third or more of patients, contributes to upper airway obstruction.[5,10] Airway clearance is impacted by decreased effectiveness of cough.[11]

Pulmonary function tests can be used to characterize abnormalities in patients with DMD, monitor progression of disease, and guide the institution of interventional strategies. $[3,4,7,8,10,12-14]$ For instance, daytime hypercapnia is detected late in the course of disease, while nocturnal hypercapnia occurs earlier and can be an indication for noninvasive intermittent positive pressure ventilation. $[4,8,9]$ Forced vital capacity (FVC), forced expiratory volume in 1 second (FEV1), and peak expiratory flow (PEF) decrease with progression of disease when reported as a percentage of predicted value.[3,4,7,13-16]

Golden retriever muscular dystrophy (GRMD) is the closest genotypic and phenotypic animal model of DMD.[17-21] Progressive skeletal muscle atrophy, contracture, abnormal gait, and exercise intolerance occur, with signs beginning shortly after birth.[22-25] As in people, death may occur due to respiratory failure.[19,23,26,27] Some puppies develop a socalled fulminant form of the disease that can lead to death in the neonatal period.[19] Valentine, et al. speculated that death could occur due to early, severe diaphragmatic involvement.[19] In our experience, these severely affected pups often have dramatic respiratory distress, in keeping with respiratory muscle disease, potentially compounded by delayed pulmonary development (pulmonary hypoplasia), akin to that seen with congenital diaphragmatic hernia.[28](Kornegay JN, McLean S, unpublished observations, 2011) As GRMD dogs mature, they continue to have respiratory difficulty, as evidenced most notably by increased respiratory rate and abdominal breathing.[19] Dogs exhibit macroglossia, drooling, and difficulty eating.[22-25] Pharyngeal and esophageal muscles may be affected. [22-25,29]. Associated difficulty in swallowing and regurgitation can predispose GRMD dogs to aspiration, with $60 \%$ reportedly developing pneumonia in one study.[27] Evaluation of the pulmonary system in GRMD dogs has been limited to clinical and radiographic assessment. Radiographic abnormalities include diaphragmatic flattening and undulations, hyperinflation of the lung, and hiatal hernia.[29,30]

The value of GRMD as a model for DMD would be greatly enhanced with practical tools to monitor progression of respiratory dysfunction during treatment trials. In our study, arterial blood gas analysis, tidal breathing spirometry, and respiratory inductance plethysmography (RIP) were performed to determine if quantifiable abnormalities could be identified in unsedated, untrained, GRMD dogs. We hypothesized that GRMD dogs would have: increased partial pressure of arterial $\mathrm{CO}_{2}\left(\mathrm{PaCO}_{2}\right)$ and decreased tidal volume (TV), as a result of hypoventilation; decreased peak tidal inspiratory flow (PTIF) and decreased expiratory time relative to inspiratory time (TimeExp:TimeInsp), as a result of upper airway obstruction and relatively prolonged inspiration; and asynchronous chest and abdominal movements, as a result of diaphragm weakness. 


\section{Materials and Methods}

\subsection{Animals and instrumentation}

Golden retriever-cross dogs from a GRMD colony were studied. Eleven dogs were affected with GRMD and eleven dogs were phenotypically normal, heterozygote carriers (control dogs). Dogs from both groups were used in breeding. Newborn GRMD dogs were identified based on elevation of serum creatine kinase (CK) and subsequently developed characteristic clinical signs. In cases where CK results were ambiguous, genotype was confirmed by polymerase chain reaction (PCR) methodology as previously described.[31] Dogs were used and cared for according to principles outlined in the National Institutes of Health Guide for the Care and Use of Laboratory Animals. Experimental procedures were approved by the Institutional Animal Care and Use Committee of the University of North Carolina.

Pairs of dogs between groups were age-matched within 2.5 months, and were from the same litter when possible. No dog had a history of illness in the month prior to study. Immediately prior to data collection, all dogs had a general physical examination including thoracic auscultation. No dogs had abnormal lung sounds on auscultation nor increased respiratory effort. Two GRMD dogs were noted to have intermittent stertorous breathing. One affected dog had a heart murmur on auscultation. One of the control dogs had mildly elevated body temperature initially; however, it was normal on repeat measurement.

Dogs with GRMD had postural changes consistent with a mild disease phenotype.[23,32] All affected dogs had some degree of macroglossia. In six of the dogs, the macroglossia was moderate to severe, with some of these dogs unable to fully close their mouth due to the size of their tongue.

Dogs were not trained to the equipment or procedures. All data collection was performed with the dog on an examination table in a quiet room without distractions. Dogs were instrumented for RIP with a commercially available, telemetric, jacketed system (Data Sciences International, St. Paul, MN) prior to beginning any data collection. Rib cage and abdominal elastic RIP bands were incorporated within a spandex shirt, and were placed so that 2 fingers could pass, with minimal pressure, between the bands and the body wall to avoid restriction of breathing. A loose fitting outer mesh jacket held wires and a telemetric device, which transmitted data to the processing computer.

\subsection{Arterial blood gas analysis}

Arterial blood samples were collected from the dorsal pedal artery using syringes containing dry lithium heparin (Vital Signs Colorado, Inc., Englewood, CO). Samples were immediately analyzed using a bed-side instrument, with values adjusted for body temperature by the device (VetScan i-STAT, Abaxis, Union City, CA). Values obtained included: partial pressure of oxygen $\left(\mathrm{PaO}_{2}\right)$, partial pressure of carbon dioxide $\left(\mathrm{PaCO}_{2}\right)$, acidity $(\mathrm{pH})$, bicarbonate concentration $\left(\mathrm{HCO}_{3}\right)$, carbon dioxide content $\left(\mathrm{TCO}_{2}\right)$, and lactate concentration. For dogs with a $\mathrm{PaO}_{2}<80 \mathrm{mmHg}$, a second specimen was obtained to minimize the possibility of venous contamination of the initial specimen resulting in the low $\mathrm{PaO}_{2}$. The specimen with the higher $\mathrm{PaO}_{2}$ value was used.

\subsection{Tidal breathing spirometry}

After blood collection, dogs were allowed to acclimate on an examination table for at least 5 minutes, until they maintained a stable, standing or sternal position, with minimal physical restraint. A clear, rigid, plastic, anesthetic facemask with a rubber gasket (Surgivet, Smiths Medical ASD, Inc., St. Paul, MN) was placed over the dog's muzzle and secured via a strap behind the ears. The same size facemask was used in all dogs and was sufficient to cover the 
commissures of the lips, while minimizing dead space. The mask was attached to a pneumotachograph (Model 3700, Hans Rudolph, Inc., Shawnee, KS), which was connected to a pressure transducer (DP45, Validyne, Northridge, CA). The signal was digitized, stored, and analyzed using a commercial physiology software platform (Ponemah Physiology Platform 4.90 - SP2, Data Sciences International, St. Paul, MN), with a sampling rate of 500 Hz. A capnometer (Welch Allyn, Inc., Skaneateles Falls, NY) was placed distal to the pneumotachograph. The pneumotachograph was calibrated using an inline flow meter (TSI, Shoreview, MN) prior to data collection from each dog. Data were collected for a minimum of 4 minutes.

Throughout collection of spirometric data, end-tidal $\mathrm{CO}_{2}\left(\mathrm{etCO}_{2}\right)$ was monitored continuously and respiratory rates were measured by visual assessment each minute. Values for etCO $\mathrm{C}_{2}$ were recorded for most dogs at 1,2,3, and 4 minutes. The mask was to be removed if the dog became agitated or appeared distressed, or if either the etCO $\mathrm{CO}_{2}$ or measured respiratory rate showed steadily increasing values. The mask was to be replaced as soon as the dog appeared to relax and was breathing with ease.

Spirometry data were analyzed as has been previously reported.[33-38] For each dog, 8-10 tidal breaths representative of the predominant pattern were selected. Criteria for selection of breaths included: lack of artifacts, such as resulting from panting, sniffing, cough, or movement, on visual examination of the flow-time tracings; a difference of $\mathbf{5} \%$ between inspiratory and expiratory volumes, to exclude breaths with evidence of leaking of air from the mask; and, a respiratory frequency of $\mathbf{5 0}$ breaths per minute, to exclude panting. Quantitative parameters included: respiratory rate (RR), expiratory time (TimeExp), inspiratory time (TimeInsp), tidal volume (TV), minute volume (MV), peak tidal expiratory flow (PTEF), peak tidal inspiratory flow (PTIF), tidal expiratory flow at 50\%, 25\% and $12.5 \%$ of TV (TEF50, TEF25, TEF12.5), and tidal inspiratory flow at 50\% of TV (TIF50). Tidal volume, PTEF and PTIF were also compared after adjusting for body weight (TV/kg, $\mathrm{PTEF} / \mathrm{kg}$, and PTIF/kg). Ratios were determined for: expiratory versus inspiratory time and flows (TimeExp:TimeInsp, PTEF:PTIF, TEF50:TIF50); inspiratory flows at different volumes of the same breath (PTIF:TIF50); and expiratory flows at different volumes of the same breath (PTEF:TEF50, PTEF:TEF25; PTEF:TEF12.5; TEF50:TEF25; TEF50:TEF12.5; TEF25:TEF12.5). Flow-volume curves were subjectively described.

\subsection{Respiratory inductance plethysmography}

Immediately following spirometry, the facemask was removed and the dogs were allowed to rest for 5 minutes. RIP data were then collected for 5 minutes without the facemask in place, and an additional 5 minutes with the facemask placed over the muzzle as described for spirometry. Voltage signals from the abdominal and rib cage bands were collected, stored, and analyzed using a commercial physiology software platform (Ponemah Physiology Platform 4.90 - SP2, Data Sciences International, St. Paul, MN). Signals were calibrated for volume for each dog using qualitative diagnostic calibration (QDC) and a fixed volume.[39] The median tidal volume obtained by spirometry for each dog was used for this volume calibration, as the processor did not have the capability to concurrently collect spirometry and RIP data. To obtain the best match of breathing mechanics during the periods of spirometry and RIP data collection, the last 5 minutes of RIP data collected with the facemask in place were used for statistical analysis. For each dog, at least 8 breaths representative of the predominant pattern were selected. Quantitative parameters included: RR, TimeExp, TimeInsp, TV, MV, PTEF, PTIF, TEF50, TIF50, and phase angle. Ratios were determined for: TimeExp:TimeInsp, PTEF:PTIF, and TEF50:TIF50.

The relationship between rib cage and abdominal motion was examined quantitatively by measurement of phase angle. Phase angle was calculated by the software program as 360 
times the time difference between peak chest and abdominal motion divided by the respiratory cycle time and reported in degrees. Qualitative evaluation was done by the visual comparison of the raw voltage output from the rib cage and abdomen bands over time and as Konno-Mead plots. The raw signals were not standardized for gain or calibrated by volume. Konno-Mead plots display output from the abdomen band on the $\mathrm{x}$-axis and that from the rib cage band on the y-axis, and typical patterns can be recognized.[40,41] In normal breathing, the rib cage and abdomen expand and contract in synchrony and the Konno-Mead plot will be a closed loop with a positive slope. In asynchronous breathing, the motion of the rib cage does not occur at the same time as the abdomen and the resulting plot will be a more open loop. In paradoxical breathing, the abdomen decreases in volume as the rib cage expands and the abdomen expands as the rib cage decreases in volume. The resulting plot will have a negative slope.

\subsection{Statistical analysis}

Statistical analyses were conducted with SigmaPlot software version 11.0.(Systat Software, Inc, Chicago, IL). Coefficients of variation (one standard deviation as a percentage of the mean) were calculated for spirometric data from each dog as an indication of consistency of measurements between their representative breaths.[33] Student's $t$-test or Mann-Whitney rank sum test was used to compare data from GRMD and control dogs. Pearson productmoment correlation or Spearman rank order was used to identify any relationship between $\mathrm{RR}$ and $\mathrm{PaCO}_{2}$ and to compare parameters obtained by spirometry with those obtained by RIP. P $<0.05$ was considered significant. For consistency, data are reported as median (25-/ 75-percentiles).

\section{Results}

\subsection{Subjects, respiratory rates and heart rates}

The initial study group was comprised of 11 GRMD and 11 control dogs. Spirometric data were inadequate for analysis from one of the eleven GRMD dogs. This dog maintained his general body position throughout collection, but made frequent movements of his head. During analysis of the tracings, eight representative breaths could not be identified based on the defined criteria. As such, this dog and the age-matched control dog were removed from all analysis, leaving $10 \mathrm{dogs}$ in each of the 2 groups.

Eight of the remaining 10 pairs of dogs were littermates. The median (25-/75- percentile) age of GRMD dogs was 47.7 (17.0/55.4) months, and was purposefully similar to control dogs, which had a median age of $47.5(17.0 / 53.4)$ months. Nine of the 10 GRMD dogs were male, and all control dogs were female. Dogs with GRMD weighed less (16.5 [15.3/18.9] kg) than control dogs (20.3 [19.0/22.7] kg, P=0.001). On physical examination, GRMD dogs had a lower respiratory rate (20 [16/24] breaths per minute) than control dogs (28 [24/36] breaths/ $\min , \mathrm{P}=0.027)$. In contrast, dogs with GRMD had a higher heart rate $(131[128 / 144]$ beats/ $\mathrm{min})$ compared with control dogs (120 [108/136] beats/min), but statistical significance was not reached $(\mathrm{P}=0.079)$.

\subsection{Performance of procedures}

Arterial blood specimens were obtained without difficulty from all 22 dogs. A single puncture was required for successful specimen collection in 19 dogs. Based on our criteria to collect a second specimen if the $\mathrm{PaO}_{2}$ was $<80 \mathrm{mmHg}$, a second specimen was collected in 3 dogs.

Spirometry data were successfully collected from 21 of 22 dogs (95\%). The majority of dogs $(n=19)$ were evaluated while sternal, with 2 GRMD dogs and 1 control dog preferring to 
stand. The facemask had to be removed from 5 of 22 dogs (22\%) when the dogs either would not remain in position with minimal restraint or showed signs of respiratory difficulty. Of these $5 \mathrm{dogs}, 3$ had GRMD and 2 were control dogs. The mask was removed once in $3 \mathrm{dogs}$, twice in $1 \mathrm{dog}$, and three times in $1 \mathrm{dog}$. The mask could be replaced within approximately one to two minutes, and data collection continued. Mask removal was most commonly associated with dog resistance to the mask or panting, which would preclude quality data collection. Removal was only associated with apparent respiratory difficulty in 1 GRMD dog. None of the dogs were noted to have increased etCO $\mathrm{C}_{2}$, indicating that the instrumentation was not creating a large dead space. Analysis of recorded etCO measurements at 1 and 4 minutes of data collection failed to show any difference when comparing: values at 1 minute and 4 minutes for each dog using paired t-test $(\mathrm{P}=0.529)$; or the difference between the two values between GRMD dogs and controls $(\mathrm{P}=0.569)$.

Respiratory inductance plethysmography was successfully performed in 18 of 20 (90\%) dogs. There were insufficient breaths with a regular pattern to analyze due to movement artifact in one GRMD and one control dog. These dogs were removed from analysis. In another dog with GRMD, abnormal abdominal motion interfered with the analysis software which made the derived data inaccurate. Data from this dog was excluded from numerical comparisons, but not from qualitative assessment.

\subsection{Arterial blood gas analysis}

Dogs with GRMD had higher $\mathrm{PaCO}_{2}$ (median [25-/75- percentile], 38.8 [35.3/41.8] mmHg) compared with control dogs (35.1 [31.9/36.2] mmHg; $\mathrm{P}=0.014)$. In addition, GRMD dogs had higher $\mathrm{HCO}_{3}(23.6$ [21.1/24.0] mEq/L; control dogs, 19.9 [18.4/21.1] mEq/L; $\mathrm{P}<0.001)$, and $\mathrm{TCO}_{2}(25.0$ [21.8/25.0] mEq/L; control dogs, 21.0 [19.0/22.3] mEq/L; $\mathrm{P}=0.001)$. No differences were found for $\mathrm{PaO}_{2}(\mathrm{P}=0.979), \mathrm{pH}(\mathrm{P}=0.762)$, or lactate concentration $(\mathrm{P}=0.571)$. Correlation statistical analysis failed to identify a relationship between $\mathrm{RR}$ as measured during physical examination or by spirometry, and $\mathrm{PaCO}_{2}$.

\subsection{Tidal breathing spirometry}

Results of tidal breathing spirometry are summarized in Table 1. Dogs with GRMD had significantly higher peak tidal expiratory flow as measured directly (PTEF, $\mathrm{P}=0.002$ ) and when compared with peak tidal inspiratory flow (PTEF:PTIF, $\mathrm{P}<0.001$ ). Tidal expiratory flows at $50 \%$ of TV (TEF50) were also significantly elevated in the GRMD group $(\mathrm{P}=0.002)$. Ratios of flow values early in expiration to values later in expiration were elevated in GRMD dogs (PTEF:TEF25, $\mathrm{P}=0.002$; PTEF:TEF12.5, $\mathrm{P}<0.001$; TEF50:TEF25; $\mathrm{P}<0.001$; TEF50:TEF12.5, $\mathrm{P}<0.001$; TEF25:TEF 12.5 $\mathrm{P}=0.002$ ), with the exception of PTEF:TEF50 ( $\mathrm{P}=0.970)$.

Coefficients of variation for directly measured parameters (i.e. not including ratios) for each dog ranged from 2-33\%. Mean coefficients of variation from all dogs for each measured parameter ranged from $7 \%$ for TV to $20 \%$ for EF12.5. Mean coefficients of variation from all dogs were similar between groups for most measured parameters, with the mean value more often lower for GRMD dogs than controls.

The increased expiratory flows and ratios were apparent in the predominant shapes of the flow-volume loops as assessed subjectively. In 9 of 10 GRMD dogs, the expiratory phase of the flow-volume loop was visibly greater than the inspiratory phase, and a pronounced peak expiratory flow was often present (Figure 1). 


\subsection{Respiratory inductance plethysmography}

Good correlation was found between parameters measured by both spirometry and RIP, which included TV, BPM, TimeExp, TimeInsp, PTIF, PTEF, TIF50, and TEF50 ( $\mathrm{P}<0.001)$, although absolute values differed between methods. Similar to spirometry, dogs with GRMD had significantly greater PTEF ( $\mathrm{P}=0.008)$, TEF50 ( $\mathrm{P}<0.001)$, PTEF:PTIF ( $\mathrm{P}=0.004)$ and TEF50:TIF50 $(\mathrm{P}<0.001)$. Other parameters, including phase angle, were not different between groups (Table 2).

Abnormal abdominal motion by qualitative analysis was present in 7 of 10 (70\%) GRMD dogs. Near synchronous motion of the rib cage and abdomen occurred during expansion of the rib cage, but paradoxical abdominal motion occurred in early inspiration and/or late expiration. This resulted in two abdominal peaks for each expansion of the rib cage. In comparison to the expected abdominal peak during inspiration, the size of the abnormal abdominal peak ranged from minimal to marked (Figure 2). In these dogs, the Konno-Mead plots formed a " $\mathrm{C}$ " shape arising from the paradoxical motion at the beginning of inspiration and end of expiration (Figure 2). The failure to identify a difference in phase angle between the two groups of dogs is a result of the calculation method, which accounts only for the relationship of peak rib cage expansion and predominant abdominal peak. No control dogs exhibited this abnormal abdominal motion and Konno-Mead plots were roughly linear with a positive slope. No obvious relationship could be seen between the degree of Konno-Mead plot abnormality and age, body weight, $\mathrm{CO}_{2}, \mathrm{TV}, \mathrm{PEF}$, or PEF:PIF values.

Our primary purposes for collecting RIP data were to compare results with spirometry data and to assess rib cage and abdominal motion. Due to the inability to collect spirometry and RIP data simultaneously, we focused our quantitative assessment on RIP with a facemask in place to have consistency of breaths. However, to investigate the unusual abdominal breathing pattern further, we qualitatively examined the RIP data from the period when the dogs were not wearing a facemask. The quality of these RIP tracings was generally poor as a result of artifacts from body movement and panting. Better results would likely be obtained if dogs were left alone in a kennel in a quiet room for prolonged periods of time. This is a feasible future approach given the telemetric capabilities of the system. Despite the limitation in the data collected without a facemask, 3 GRMD dogs and no control dogs had detectable periods of paradoxical breathing (i.e. greater abnormal abdominal motion) without a facemask in place (Figure 2).

\section{Discussion}

Respiratory function tests used in this study successfully identified abnormalities in dogs with GRMD without sedation or training. Higher peak expiratory flows were documented by spirometry and RIP. Abnormal abdominal motion was identified by RIP in the majority of dogs. Moreover, relatively higher $\mathrm{PaCO}_{2}$ and $\mathrm{HCO}_{3}$ concentrations were found by arterial blood gas analysis.

The median value of the PTEF in the GRMD dogs was over $80 \%$ greater than that of control dogs, based on spirometry and RIP. Flow values as calculated from RIP measurements were not identical to those measured by spirometry, but showed good correlation. Discrepancies were expected because data were not collected simultaneously and different methodologies were used.[42] Increased expiratory flows have not been reported in people with DMD as measured with forced maneuvers. One plausible explanation for the increased peak tidal expiratory flows in GRMD dogs is increased elastic recoil secondary to hyperinflation. Hyperinflation is a common radiographic finding in GRMD dogs.[29,30] Purposeful hyperinflation in healthy people causes increased forced peak expiratory flows,[43] and is a technique used by people with DMD to improve effectiveness of cough through increased 
cough peak flows.[44] With hyperinflation due to lower airway obstruction, chest wall recoil may be inwards, adding to lung recoil in driving tidal expiratory flow.[45] Such an effect could be amplified in GRMD dogs because of replacement of intercostal muscles with fibrous tissue.

The genesis of the hyperinflation in GRMD dogs is not known, and the study design did not include evaluation for hyperinflation. Brummit, et al. hypothesized that hyperinflation might be secondary to decreased diaphragmatic excursion due to pseudohypertrophy of the diaphragm, leading to a compensatory change in intercostal musculature.[30] Another possibility is that bronchial smooth muscle undergoes hyperplastic and/or hypertrophic changes as is seen in GRMD skeletal muscle,[46] resulting in lower airway obstruction and subsequent air trapping. Although striated muscle, rather than smooth muscle, is characteristically described as being affected by dystrophin deficiency, dystrophin has been identified in human airway smooth muscle.[47] Further, two dogs with German short-haired pointer muscular dystrophy and radiographic evidence of hyperinflation were reported to have abnormally increased expiratory efforts, consistent with obstructive lower airway disease.[48]

The expected spirometric abnormality in people with obstructive lower airway disease during forced exhalation is a decrease in peak expiratory flow, the opposite of the finding in these GRMD dogs. However, increased tidal breathing peak expiratory flow has been reported in horses with recurrent airway obstruction (heaves).[37,38] Horses in the study by Petsche, et al. also showed increased ratios of expiratory flows compared with expiratory flows later in expiration, as found in GRMD dogs.[37] Dogs with chronic bronchitis were reported to have greater PTEF:EF25 compared with control dogs, also consistent with our findings.[49] These differences in reported expiratory flows between animals and people might be due to the measurement of maximal expiratory efforts in people and of tidal breathing in dogs and horses. Purposeful, forced exhalation might obscure the relatively smaller pressures generated by elastic recoil.

Increased time spent in expiration is another indication of obstructive airway disease. Respiratory rate must be considered when comparing expiratory times between groups, so ratios such as expiratory time to inspiratory time are used. In GRMD dogs the ratio of expiratory time to inspiratory time was greater than that of control dogs. Although not statistically significant, the $\mathrm{P}$ value was $<0.1$ for spirometric data. As such, a difference might be found with a larger study population.

Other factors besides recoil can influence peak expiratory flow. Elevated respiratory rates in dogs[50] and increased body size in people have been associated with increased expiratory flows. Study dogs with GRMD had slightly lower respiratory rates compared with controls, though the difference between groups based on spirometric data was not statistically significant. While study dogs with GRMD were of lower body weight than control dogs, body weight differences between these populations of dogs might not reflect thoracic volume changes because of the influence of muscle atrophy on body weight. Regardless, differences in peak tidal expiratory flow persisted when values were divided by body weight.

The RIP bands and shirts might have decreased chest wall compliance, causing a restrictive breathing pattern. However, care was taken during their application to avoid interference with normal respiration. Although not directly comparable, a study by Rozanski, et al. failed to find an effect of chest bandaging on functional residual capacity in dogs that were standing or in sternal recumbency.[51] Most importantly, in our study, instrumentation of dogs for RIP was identical between groups. 
Finally, an increase in peak expiratory flow can be found in tidal breathing spirometry in dogs with restrictive disease.[52] With this said, the GRMD dogs failed to exhibit the expected increased respiratory rates, decreased tidal volumes, or increased peak inspiratory flows typical of restrictive disease. Further, although people with DMD are considered to have a restrictive lung pattern of breathing, increased forced expiratory flows are not reported in these patients. Surprisingly, GRMD dogs had a slower respiratory rate than control dogs. Although this might reflect less anxiety due, potentially, to more frequent handling of affected dogs, the heart rates of GRMD dogs were not slower than control dogs. Importantly, as with other observations, data must be interpreted in the context of the mild disease phenotype of the GRMD dogs studied. More severely affected dogs might have a different pattern of disease.

An abnormal asynchronous breathing pattern, characterized by two expansions of the abdomen for each expansion of the rib cage, was present in the majority of dogs with GRMD. This pattern has been reported in some people with chronic obstructive pulmonary disease (COPD), lending support to the possibility of airway obstruction and hyperinflation contributing to respiratory dysfunction in these dogs.[53] This pattern was described by Ashutosh, et al.[53] as the abdomen signal moving abruptly downward near or at the end of inspiration and then moving upward later in expiration, with continued upward movement during a variable part of the expiration.[53] An EMG of the diaphragm showed no activity during expiration, indicating the second expansion of the abdomen was passive.[53] Further, a few GRMD dogs demonstrated a greater degree of abnormal abdominal breathing with the facemask off, which is more consistent with COPD than upper airway obstruction.

Hyperinflation resulting in displacement of the diaphragm and diaphragm fatigue have been proposed as mechanisms contributing to paradoxical diaphragm movements in people with COPD and emphysema.[53,54]

Lo Mauro, et al., did not report this double abdominal motion in people with DMD.[15] Rather, they noted that the abdominal contribution to tidal volume progressively declined in DMD patients. Our instrumentation did not allow accurate determination of the relative percentage contributions of abdomen and rib cage to tidal volume. Preliminary results of RIP of GRMD dogs from the same colony of dogs as those reported here demonstrated an increase in phase angle in dogs with GRMD, indicating a loss of synchrony between peak rib cage and peak abdominal motion.[55] Phase angles were normal in most GRMD dogs in our study because the first abdominal deflection was relatively synchronous with that of the rib cage, even though the second deflection was paradoxical. Explanations for differences in results between these study populations include: age of dogs, with our dogs being older; phenotypic variation with our dogs likely being less severely affected; and, that our dogs were wearing facemasks at the time of RIP. Facemasks were used in our dogs because we were not able to measure airflow by spirometry concurrently with RIP. For volume calibration of RIP signals, tidal volume measured by spirometry was used. As breathing through a mouthpiece is known to alter breathing patterns in people, we chose to perform RIP with the mask in place for consistency with breathing during spirometry. Abnormal abdominal motion was common to both studies and varied among affected dogs. This finding suggests that RIP can be used to monitor disease progression over time. Additional studies are needed to further substantiate these findings.

Evidence of hypoventilation was identified by arterial blood gas analysis, but not by tidal breathing spirometry. Dogs with GRMD had higher $\mathrm{PaCO}_{2}$ and $\mathrm{HCO}_{3}$ concentrations compared with control dogs. None of the dogs had a $\mathrm{PaCO}_{2}$ greater than $45 \mathrm{mmHg}$, the maximum value of the reference range provided by the manufacturer. This absence of absolute hypercapnia is consistent with findings in people with DMD, where daytime elevations are not seen until terminal stages of disease.[3] The finding of increased $\mathrm{PaCO}_{2}$ in 
GRMD dogs is confounded by the difference in respiratory rates between GRMD dogs and controls. Dogs with GRMD had a lower median respiratory rate during physical examination than control dogs, which could contribute to an increase in $\mathrm{PaCO}_{2}$. However, there was no statistical difference in minute volume or respiratory rates between groups as measured by spirometry, and there was no statistical correlation between $\mathrm{PaCO}_{2}$ and respiratory rate measured during physical examination or spirometry. The concurrent finding of increased $\mathrm{HCO}_{3}$ in GRMD dogs could indicate a compensatory response to a more chronic increase in $\mathrm{PaCO}_{2}$ that would not be influenced by respiratory rate. The best applications for arterial blood gas analysis in GRMD might be for monitoring individual dogs, using their baseline value as the control, or for assessment of pulmonary function in more advanced stages of the disease.

Tidal breathing spirometry did not show evidence of hypoventilation or upper airway obstruction in GRMD dogs, as hypothesized. Tidal breathing flow-volume loops in upper airway obstruction of infants[56,57] and dogs[33-36,58] show characteristic flattening of the inspiratory limb. Although the shape of the flow-volume loops from GRMD dogs was consistent with upper airway obstruction, the expiration limb was consistently abnormal rather than the inspiratory limb, when compared with the control dog population. The abnormality in expiration was confirmed by the independent RIP results. Tidal breathing spirometry is inherently less sensitive in detecting obstruction than studies performed with forced breathing efforts. While dogs cannot be made to voluntarily expire maximally, sensitivity of tidal breathing spirometry might be improved by inducing increased breathing efforts. Exercise is not a ready solution because GRMD dogs are often reluctant to exert themselves. Also, a lack of consistency in effort of exertion between dogs could confound results. Systemic administration of doxapram, a respiratory stimulant, or inhalation of $10 \%$ $\mathrm{CO}_{2}$ during spirometry, might improve sensitivity.[59] Further investigation is warranted.

In addition to potential confounding factors described above, there are other limitations to this study. Importantly, the GRMD dogs of this study were mostly breeding males with an overall mild disease phenotype. More severely affected GRMD dogs included in preclinical studies might have more pronounced changes that could be tracked in response to treatment. Serial monitoring of dogs, or testing of dogs with more severe disease, might have uncovered additional abnormalities suitable for monitoring. Moreover, given the nature of the study, we did not systematically collect additional phenotypic data with which to correlate the respiratory dysfunction. Occult pneumonia in these dogs could have influenced results, but would be an unlikely cause of increased peak expiratory flow. Control dogs were carriers of GRMD and could have mild abnormalities.[24,60,61] Using these dogs as controls had the benefit of using age-matched, and often litter-matched, dogs of the same breed for comparison. A study by Amis, et al. found differences in tidal-breathing flow volume loops in golden retriever dogs compared with other breeds.[33] Radiographic or histologic evaluation of the lungs of the study dogs might have identified hyperinflation or bronchial involvement. Unfortunately, we were limited to the reported procedures in this study.

This study is the first to assess respiratory function of dogs with muscular dystrophy using blood gas analysis, tidal breathing spirometry, and RIP, and compliments an earlier abstract reporting results from RIP in dogs from this same colony.[55] The methods evaluated were specifically chosen to avoid sedation, in anticipation of their use for repeated monitoring in future studies, as sedation has the potential to affect measured results as well as influence disease progression. The testing methods were successfully performed and provided specific parameters for monitoring. However, the loss of data from even a few dogs is not ideal, particularly when many treatment studies are limited to small subject numbers. Success of data collection could be improved by having data analysis performed at the time of, or 
shortly after, collection. In our study, data were collected from all dogs prior to analysis, after which the dogs were no longer available for repeated study. Initial training and acclimatization of dogs to the procedures might also improve overall data quality. Such training would be necessary for studies with repeated measures because dogs will become acclimated through the repeat testing, and this change in degree of stress could influence results. Spirometry, in particular, requires a compliant subject. Struggling against the mask will result in leaking around the diaphragm and invalid results. Interestingly, the facemask improved the quality of tracings from RIP. Decreased panting and body movement artifact during RIP without a facemask in place could also be achieved by prolonged instrumentation. Performance of RIP in dogs without a facemask is probably best done with the dog alone in a kennel, in a quiet room. Such an approach is feasible through the telemetric capabilities of the RIP system used. For both spirometry and RIP, identification of more subtle abnormalities might be possible with greater numbers of analyzable breaths.

In summary, this study demonstrated the successful application of arterial blood gas analysis, tidal breathing spirometry, and RIP for the monitoring of dogs with GRMD without sedation. Further, we found that GRMD dogs have increased peak tidal expiratory flows, which might be the result of hyperinflation, relative hypercapnia, and abnormal abdominal breathing patterns. Additional study is warranted to investigate the underlying pathophysiology of these findings.

\section{Acknowledgments}

Support for care of study dogs came from 1U24NS059696-01A1 (NINDS), Co-operative Program in Translational Research: Proposal for Establishment of the National Center for Canine Models of Duchenne Muscular Dystrophy (NCDMD) (to JNK) and Muscular Dystrophy Association Infrastructure Grant to the Translational Research Advisory Committee (TRAC) (to JNK). A supplement to 1U24NS059696-01A1 was used to purchase spirometry equipment used in these studies.

The authors are grateful for the support of Janice A. Dye, DVM, PhD, Dipl ACVIM (SAIM) for careful review of our data and manuscript.

Funding for this project was provided through a supplement to Co-operative Program in Translational Research: Proposal for Establishment of the National Center for Canine Models of Duchenne Muscular Dystrophy (NCDMD). NIH (NINDS/NIAMS) (1U24NS059696-01A1)

\section{References}

[1]. Cowan J, Macdessi J, Stark A, Morgan G. Incidence of Duchenne muscular dystrophy in New South Wales and Australian Capital Territory. J Med Genet. 1980; 17:245-249. [PubMed: 7205898]

[2]. Emery AE. Population frequencies of inherited neuromuscular diseases--a world survey. Neuromuscul Disord. 1991; 1:19-29. [PubMed: 1822774]

[3]. Inkley SR, Oldenburg FC, Vignos PJ Jr. Pulmonary function in Duchenne muscular dystrophy related to stage of disease. Am J Med. 1974; 56:297-306. [PubMed: 4813648]

[4]. Laghi F, Tobin MJ. Disorders of the respiratory muscles. Am J Respir Crit Care Med. 2003; 168:10-48. [PubMed: 12826594]

[5]. Gozal D. Pulmonary manifestations of neuromuscular disease with special reference to Duchenne muscular dystrophy and spinal muscular atrophy. Pediatr Pulmonol. 2000; 29:141-150. [PubMed: 10639205]

[6]. McCool FD, Mayewski RF, Shayne DS, Gibson CJ, Griggs RC, Hyde RW. Intermittent positive pressure breathing in patients with respiratory muscle weakness. Alterations in total respiratory system compliance. Chest. 1986; 90:546-552. [PubMed: 3530648]

[7]. Rideau Y, Jankowski LW, Grellet J. Respiratory function in the muscular dystrophies. Muscle Nerve. 1981; 4:155-164. [PubMed: 7207506] 
[8]. Bushby K, Finkel R, Birnkrant DJ, et al. Diagnosis and management of Duchenne muscular dystrophy, part 2: implementation of multidisciplinary care. Lancet Neurol. 2010; 9:177-189. [PubMed: 19945914]

[9]. Toussaint M, Chatwin M, Soudon P. Mechanical ventilation in Duchenne patients with chronic respiratory insufficiency: clinical implications of 20 years published experience. Chron Respir Dis. 2007; 4:167-177. [PubMed: 17711917]

[10]. Raphael JC, Chevret S, Chastang C, Bouvet F. Randomised trial of preventive nasal ventilation in Duchenne muscular dystrophy. French Multicentre Cooperative Group on Home Mechanical Ventilation Assistance in Duchenne de Boulogne Muscular Dystrophy. Lancet. 1994; 343:1600_ 1604. [PubMed: 7911921]

[11]. Kravitz RM. Airway clearance in Duchenne muscular dystrophy. Pediatr. 2009; 123(Suppl 4):S231-S235.

[12]. Sejerson T, Bushby K. Standards of care for Duchenne muscular dystrophy: brief TREAT-NMD recommendations. Adv Exp Med Biol. 2009; 652:13-21. [PubMed: 20225016]

[13]. Samaha FJ, Buncher CR, Russman BS, et al. Pulmonary function in spinal muscular atrophy. J Child Neurol. 1994; 9:326-329. [PubMed: 7930415]

[14]. Phillips MF, Quinlivan RC, Edwards RH, Calverley PM. Changes in spirometry over time as a prognostic marker in patients with Duchenne muscular dystrophy. Am J Respir Crit Care Med. 2001; 164:2191-2194. [PubMed: 11751186]

[15]. Lo Mauro A, D’ Angelo MG, Romei M, et al. Abdominal volume contribution to tidal volume as an early indicator of respiratory impairment in Duchenne muscular dystrophy. Eur Respir J. 2010; 35:1118-1125. [PubMed: 19840972]

[16]. Tangsrud S, Petersen IL, Lodrup Carlsen KC, Carlsen KH. Lung function in children with Duchenne's muscular dystrophy. Respir Med. 2001; 95:898-903. [PubMed: 11716204]

[17]. Cooper BJ, Winand NJ, Stedman H, et al. The homologue of the Duchenne locus is defective in X-linked muscular dystrophy of dogs. Nature. 1988; 334:154-156. [PubMed: 3290691]

[18]. Valentine BA, Winand NJ, Pradhan D, Moise NS, de LA, Kornegay JN, Cooper BJ. Canine Xlinked muscular dystrophy as an animal model of Duchenne muscular dystrophy: a review. Am J Med Genet. 1992; 42:352-356. [PubMed: 1536178]

[19]. Valentine BA, Cooper BJ, deLahunta A, O’Quinn R, Blue JT. Canine X-linked muscular dystrophy. An animal model of Duchenne muscular dystrophy: clinical studies. J Neurol Sci. 1988; 88:69-81. [PubMed: 3225630]

[20]. Sharp NJ, Kornegay JN, Van Camp SD, et al. An error in dystrophin mRNA processing in golden retriever muscular dystrophy, an animal homologue of Duchenne muscular dystrophy. Genomics. 1992; 13:115-121. [PubMed: 1577476]

[21]. Banks GB, Chamberlain JS. The value of mammalian models for duchenne muscular dystrophy in developing therapeutic strategies. Curr Top Dev Biol. 2008; 84:431-453. [PubMed: 19186250]

[22]. Kornegay JN, Tuler SM, Miller DM, Levesque DC. Muscular dystrophy in a litter of golden retriever dogs. Muscle Nerve. 1988; 11:1056-1064. [PubMed: 3185600]

[23]. Kornegay JN, Bogan JR, Bogan DJ, et al. Canine models of Duchenne muscular dystrophy and their use in therapeutic strategies. Mamm Genome. 2012; 23:85-108. [PubMed: 22218699]

[24]. Shelton GD, Engvall E. Canine and feline models of human inherited muscle diseases. Neuromuscul Disord. 2005; 15:127-138. [PubMed: 15694134]

[25]. Valentine BA, Cooper BJ. Canine X-linked muscular dystrophy: selective involvement of muscles in neonatal dogs. Neuromuscul Disord. 1991; 1:31-38. [PubMed: 1840414]

[26]. Valentine BA, Cummings JF, Cooper BJ. Development of Duchenne-type cardiomyopathy. Morphologic studies in a canine model. Am J Pathol. 1989; 135:671-678. [PubMed: 2679113]

[27]. Ambrosio CE, Fadel L, Gaiad TP, Martins DS, et al. Identification of three distinguishable phenotypes in golden retriever muscular dystrophy. Genet Mol Res. 2009; 8:389-396. [PubMed: 19440974]

[28]. Keijzer R, Puri P. Congenital diaphragmatic hernia. Semin Pediatr Surg. 2010; 19:180-185. [PubMed: 20610190] 
[29]. Bedu AS, Labruyere JJ, Thibaud JL, et al. Age-related thoracic radiographic changes in golden and labrador retriever muscular dystrophy. Vet Radiol Ultrasound. 2012; 53:492-500. [PubMed: 22702494]

[30]. Brumitt JW, Essman SC, Kornegay JN, et al. Radiographic features of Golden Retriever muscular dystrophy. Vet Radiol Ultrasound. 2006; 47:574-580. [PubMed: 17153067]

[31]. Bartlett RJ, Winand NJ, Secore SL, Singer JT, Fletcher S, Wilton S, Bogan DJ, Metcalf-Bogan JR, Bartlett WT, Howell JM, Cooper BJ, Kornegay JN. Mutation segregation and rapid carrier detection of X-linked muscular dystrophy in dogs. Am J Vet Res. 1996; 57:650-654. [PubMed: 8723876]

[32]. Kornegay, JN.; Bogan, JR.; Bogan, DJ.; Childers, MK.; Grange, RW. Golden retriever muscular dystrophy (GRMD): developing and maintaining a colony and physiological functional measurements. In: Duan, D., editor. Muscle Gene Therapy: Methods and Protocols, Methods in Molecular Biology. Vol. vol 709. Humana Press; New York: 2011. p. 105-122.

[33]. Amis TC, Kurpershoek C. Tidal breathing flow-volume loop analysis for clinical assessment of airway obstruction in conscious dogs. Am J Vet Res. 1986; 47:1002-1006. [PubMed: 3717718]

[34]. Amis TC, Smith MM, Gaber CE, Kurpershoek C. Upper airway obstruction in canine laryngeal paralysis. Am J Vet Res. 1986; 47:1007-1010. [PubMed: 3717719]

[35]. Amis TC, Kurpershoek C. Pattern of breathing in brachycephalic dogs. Am J Vet Res. 1986; 47:2200-2204. [PubMed: 3777646]

[36]. Adamama-Moraitou K, Pardali D, Prassinos NN, et al. Analysis of tidal breathing flow volume loop in dogs with tracheal masses. Aust Vet J. 2010; 88:351-356. [PubMed: 20726970]

[37]. Petsche VM, Derksen FJ, Robinson NE. Tidal breathing flow-volume loops in horses with recurrent airway obstruction (heaves). Am J Vet Res. 1994; 55:885-891. [PubMed: 7978623]

[38]. Hoffman AM, Oura TJ, Riedelberger KJ, Mazan MR. Plethysmographic comparison of breathing pattern in heaves (recurrent airway obstruction) versus experimental bronchoconstriction or hyperpnea in horses. J Vet Intern Med. 2007; 21:184-192. [PubMed: 17338167]

[39]. Sackner MA, Watson H, Belsito AS, et al. Calibration of respiratory inductive plethysmograph during natural breathing. J Appl Physiol. 1989; 66:410-420. [PubMed: 2917945]

[40]. Konno K, Mead J. Measurement of the separate volume changes of rib cage and abdomen during breathing. J Appl Physiol. 1967; 22:407-422. [PubMed: 4225383]

[41]. Warren RH, Horan SM, Robertson PK. Chest wall motion in preterm infants using respiratory inductive plethysmography. Eur Respir J. 1997; 10:2295-2300. [PubMed: 9387956]

[42]. Murphy DJ, Renninger JP, Schramek D. Respiratory inductive plethysmography as a method for measuring ventilatory parameters in conscious, non-restrained dogs. J Pharmacol Toxicol Methods. 2010; 62:47-53. [PubMed: 20435149]

[43]. Eichinger M, Walterspacher S, Scholz T, et al. Lung hyperinflation: foe or friend? Eur Respir J. 2008; 32:1113-1116. [PubMed: 18827157]

[44]. Bach JR, Bianchi C, Vidigal-Lopes M, Turi S, Felisari G. Lung inflation by glossopharyngeal breathing and "air stacking" in Duchenne muscular dystrophy. Am J Phys Med Rehabil. 2007; 86:295-300. [PubMed: 17413542]

[45]. Morris MJ, Lane DJ. Tidal expiratory flow patterns in airflow obstruction. Thorax. 1981; 36:135-142. [PubMed: 7268679]

[46]. Kornegay JN, Cundiff DD, Bogan DJ, Bogan JR, Okamura CS. The cranial sartorius muscle undergoes true hypertrophy in dogs with golden retriever muscular dystrophy. Neuromuscul Disord. 2003; 13:493-500. [PubMed: 12899877]

[47]. Sharma P, Tran T, Stelmack GL, et al. Expression of the dystrophin-glycoprotein complex is a marker for human airway smooth muscle phenotype maturation. Am J Physiol Lung Cell Mol Physiol. 2008; 294:L57-L68. [PubMed: 17993586]

[48]. Olby NJ, Sharp NJ, Nghiem PE, et al. Clinical progression of X-linked muscular dystrophy in two German Shorthaired Pointers. J Am Vet Med Assoc. 2011; 238:207-212. [PubMed: 21235374]

[49]. Padrid PA, Hornof WJ, Kurpershoek CJ, Cross CE. Canine chronic bronchitis: a pathophysiologic evaluation of 18 cases. J Vet Intern Med. 1990; 4:172-180. [PubMed: 2114481] 
[50]. Amis, TC. Chronic bronchitis in dogs. In: Kirk, RW., editor. Current Veterinary Therapy IX. W.B. Saunders Company; Philadelphia: 1986. p. 306-312.

[51]. Rozanski EA, Bedenice D, Lofgren J, Abrams J, Bach J, Hoffman AM. The effect of body position, sedation, and thoracic bandaging on functional residual capacity in healthy deepchested dogs. Can J Vet Res. 2010; 74:34-39. [PubMed: 20357956]

[52]. Hoffman AM. Airway physiology and clinical function testing. Vet Clin North Am Small Anim Pract. 2007; 37:829-843. [PubMed: 17693201]

[53]. Ashutosh K, Gilbert R, Auchincloss JH Jr. Peppi D. Asynchronous breathing movements in patients with chronic obstructive pulmonary disease. Chest. 1975; 67:553-557. [PubMed: 1126193]

[54]. Suga K, Tsukuda T, Awaya H, et al. Impaired respiratory mechanics in pulmonary emphysema: evaluation with dynamic breathing MRI. J Magn Reson Imaging. 1999; 10:510-520. [PubMed: 10508317]

[55]. Mead A, Malik A, Petrov M, et al. External, non-invasive monitoring of progression of cardiorespiratory dysfunction in a canine model of DMD (abstract). Mol Ther. 2010; 18:S117S118.

[56]. Filippone M, Narne S, Pettenazzo A, Zacchello F, Baraldi E. Functional approach to infants and young children with noisy breathing: validation of pneumotachography by blinded comparison with bronchoscopy. Am J Respir Crit Care Med. 2000; 162:1795-1800. [PubMed: 11069815]

[57]. Abramson AL, Goldstein MN, Stenzler A, Steele A. The use of the tidal breathing flow volume loop in laryngotracheal disease of neonates and infants. Laryngoscope. 1982; 92:922-926. [PubMed: 7098740]

[58]. Pardali D, Adamama-Moraitou KK, Rallis TS, Raptopoulos D, Gioulekas D. Tidal breathing flow-volume loop analysis for the diagnosis and staging of tracheal collapse in dogs. J Vet Intern Med. 2010; 24:832-842. [PubMed: 20412439]

[59]. McKiernan BC, Johnson LR. Clinical pulmonary function testing in dogs and cats. Vet Clin North Am Small Anim Pract. 1992; 22:1087-1099. [PubMed: 1523782]

[60]. Kornegay JN, Sharp NJ, Bogan DJ, Van Camp SD, Metcalf JR, Schueler RO. Contraction tension and kinetics of the peroneus longus muscle in golden retriever muscular dystrophy. $\mathbf{J}$ Neurol Sci. 1994; 123:100-107. [PubMed: 8064300]

[61]. Kane AM, DeFrancesco TC, Boyle MC, et al. Cardiac structure and function in female carriers of a canine model of Duchenne muscular dystrophy. Res Vet Sci. 2013; 94:610-617. [PubMed: 23231955] 

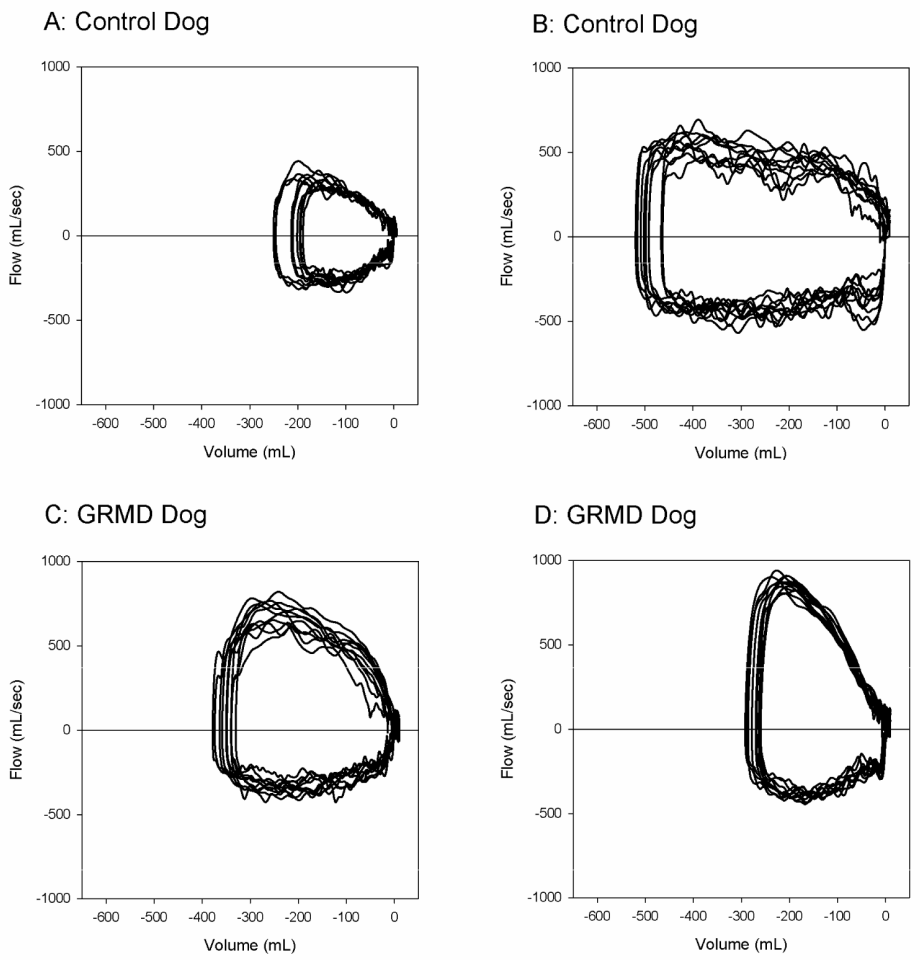

Figure 1.

Tidal breathing flow-volumes (F-V) loops of 10 representative breaths from two control dogs (A, B) and two GRMD dogs (C,D) displayed on graphs with identical scales. These dogs were selected as representative of their groups based on peak tidal expiratory flows that were closest to the median value for their group. The loops from GRMD dogs show visibly greater expiratory flows (positive values) compared with inspiratory flows (negative values) and compared with expiratory flows from control dogs. 

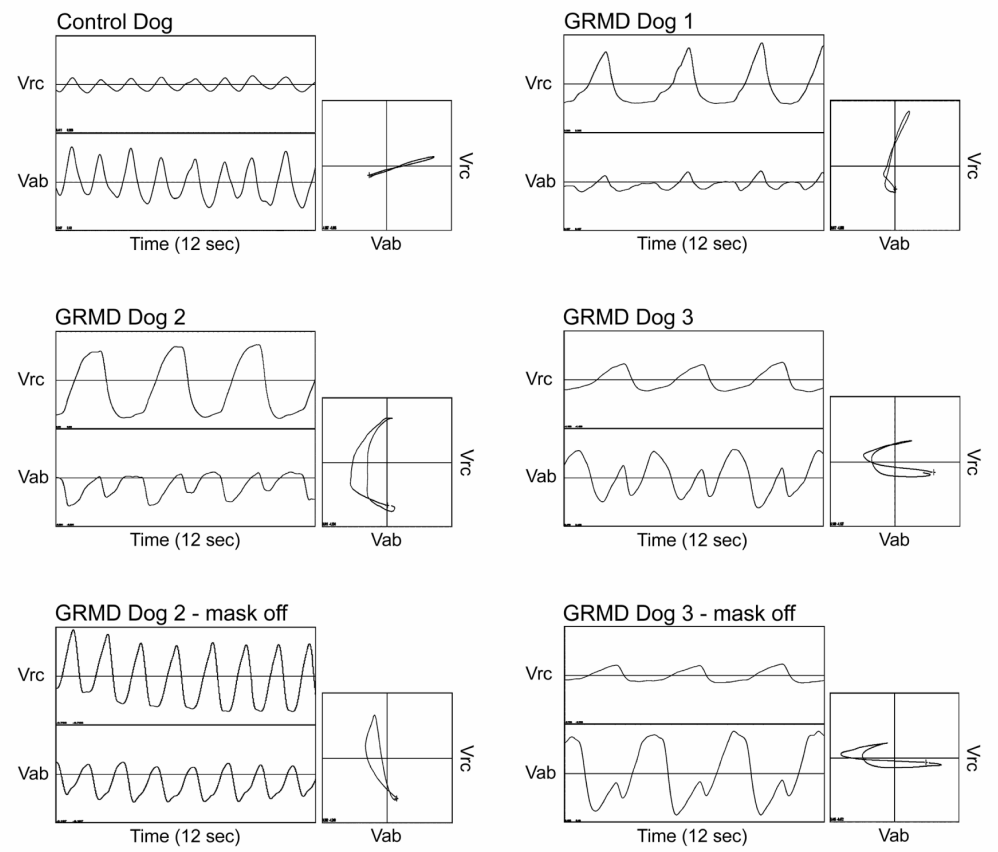

Figure 2.

Example RIP tracings and Konno-Mead plots of 1 control dog and 3 GRMD dogs. The raw output (volts) from the rib cage band (Vrc) and the adbomen band (Vab) are plotted against time in the rectangular boxes, and against each other in the square (Konno-Mead) boxes. The raw output was not calibrated or standardized. The control dog shows a synchronous pattern with coordination between rib cage and abdominal motion and the Konno-Mead plot has a positive slope. All control dogs and 3 GRMD dogs had similar patterns. GRMD Dogs 1-3 have two peaks of abdominal motion for each expansion of the rib cage. The first peak occurs at or near peak expansion of the rib cage. The second occurs during expiration. The size of the second abdominal peak is progressively larger compared with the first in these dogs. In total, five GRMD dogs had a pattern similar to GRMD Dog 1. The Konno-Mead plot shows a progressively deeper " $\mathrm{C}$ " shape in GRMD dogs 1 through 3. Tracings obtained from GRMD Dogs 2 and 3 without a face mask in place show relatively greater abnormal abdominal motion, with GRMD Dog 2 demonstrating complete paradoxical breathing. 


\section{Table 1}

Results of tidal breathing spirometry from 10 dogs with GRMD and 10 control carrier dogs expressed as median (25-/75-percentile)

\begin{tabular}{|c|c|c|c|}
\hline Value & $\begin{array}{l}\text { Control } \\
\text { Dogs }\end{array}$ & $\begin{array}{l}\text { GRMD } \\
\text { Dogs }\end{array}$ & $\begin{array}{l}P \\
\text { value }\end{array}$ \\
\hline RR (breaths/min) & $26.7(22.5 / 41.0)$ & $20.7(18.1 / 27.0)$ & 0.064 \\
\hline $\operatorname{TimeExp}(\mathrm{sec}) *$ & $1.12(0.77 / 1.38)$ & $1.57(1.20 / 1.88)$ & 0.044 \\
\hline TimeInsp (sec) & $1.10(0.75 / 1.31)$ & $1.30(0.98 / 1.69)$ & 0.098 \\
\hline TV (mL) & $356(216 / 492)$ & $366(309 / 421)$ & 0.623 \\
\hline MV (mL) & $9780(8070 / 12780)$ & $7310(6880 / 8440)$ & 0.140 \\
\hline PTEF $(\mathrm{mL} / \mathrm{sec}) *$ & $477(373 / 691)$ & $867(573 / 1086)$ & 0.002 \\
\hline PTIF (mL/sec) & $445(369 / 580)$ & $400(372 / 434)$ & 0.651 \\
\hline TEF $50(\mathrm{~mL} / \mathrm{sec}) *$ & $345(317 / 621)$ & $692(518 / 765)$ & 0.005 \\
\hline TIF $50(\mathrm{~mL} / \mathrm{sec})$ & $398(335 / 522)$ & $355(324 / 392)$ & 0.527 \\
\hline TEF $25(\mathrm{~mL} / \mathrm{sec})$ & $370(284 / 467)$ & $394(359 / 471)$ & 0.639 \\
\hline TEF $12.5(\mathrm{~mL} / \mathrm{sec}$ & $273(234 / 427)$ & $207(178 / 313)$ & 0.207 \\
\hline \multicolumn{4}{|l|}{ Weight adjusted } \\
\hline TV/kg (mL/kg) & $16.9(9.8 / 24.0)$ & $24.0(18.5 / 26.8)$ & 0.104 \\
\hline MV/kg (mL/kg) & $486(406 / 540)$ & $466(392 / 502)$ & 0.910 \\
\hline PTEF/kg (mL/sec/kg) * & $23.2(20.1 / 28.4)$ & $47.6(37.4 / 68.0)$ & $<0.001$ \\
\hline PTIF/kg (mL/sec/kg) & $21.5(17.0 / 25.3)$ & $25.5(20.6 / 26.4)$ & 0.246 \\
\hline \multicolumn{4}{|l|}{$\underline{\text { Ratios }}$} \\
\hline \multicolumn{4}{|l|}{ Expiratory:Inspiratory } \\
\hline TimeExp:TimeInsp & $1.07(0.94 / 1.10)$ & $1.24(1.01 / 1.31)$ & 0.090 \\
\hline PTEF:PTIF $*$ & $1.15(1.00 / 1.16)$ & $2.01(1.53 / 2.48)$ & $<0.001$ \\
\hline TEF50:TIF50 * & $1.00(0.91 / 1.17)$ & $1.88(1.72 / 2.30)$ & 0.002 \\
\hline \multicolumn{4}{|c|}{ Inspiratory flows:earlier inspiratory flows } \\
\hline PTIF:TIF50 & $1.11(1.09 / 1.14)$ & $1.15(1.10 / 1.19)$ & 0.241 \\
\hline \multicolumn{4}{|c|}{ Expiratory flows:later expiratory flows } \\
\hline PTEF:TEF 50 & $1.19(1.16 / 1.31)$ & $1.21(1.12 / 1.37)$ & 0.970 \\
\hline PTEF:TEF $25 *$ & $1.34(1.23 / 1.56)$ & $2.14(1.67 / 2.50)$ & 0.002 \\
\hline PTEF:TEF 12.5* & $1.69(1.46 / 2.15)$ & $4.22(3.07 / 5.07)$ & $<0.001$ \\
\hline TEF 50:TEF $25 *$ & $1.10(1.05 / 1.27)$ & $1.72(1.44 / 1.98)$ & $<0.001$ \\
\hline TEF 50:TEF $12.5 *$ & $1.43(1.26 / 1.65)$ & $3.17(2.11 / 3.67)$ & $<0.001$ \\
\hline TEF2 5:TEF $12.5 *$ & $1.25(1.20 / 1.29)$ & $1.86(1.51 / 2.99)$ & 0.002 \\
\hline
\end{tabular}

GRMD, golden retriever muscular dystrophy; RR, respiratory rate; TimeExp, expiratory time; TimeInsp, inspiratory time; TV, tidal volume; MV, minute volume; PTEF, peak tidal expiratory flow; PTIF, peak tidal inspiratory flow; TEF50, tidal expiratory flow at 50\% of TV; TIF50, tidal inspiratory flow at $50 \%$ of TV; TEF25, tidal expiratory flow at $25 \%$ of TV; TEF12.5, tidal expiratory flow at $12.5 \%$ of TV;

statistically significant difference between groups. 
Table 2

Results of respiratory inductance plethysmography from 8 dogs with GRMD and 9 control carrier dogs expressed as median (25-/75-percentile)

\begin{tabular}{|c|c|c|c|}
\hline Value & $\begin{array}{l}\text { Control } \\
\text { Dogs }\end{array}$ & $\begin{array}{l}\text { GRMD } \\
\text { Dogs }\end{array}$ & $\begin{array}{l}P \\
\text { value }\end{array}$ \\
\hline RR (breaths/min) & $31.3(25.3 / 44.5)$ & $26.9(18.8 / 41.2)$ & 0.482 \\
\hline TimeExp (sec) & $0.95(0.70 / 1.26)$ & $1.29(0.93 / 2.24)$ & 0.139 \\
\hline TimeInsp (sec) & $0.99(0.72 / 1.16)$ & $1.03(0.74 / 1.30)$ & 0.590 \\
\hline TV (mL) & $318(218 / 501)$ & $309(272 / 421)$ & 0.896 \\
\hline MV (mL) & $9771(7183 / 13273)$ & $8871(6273 / 14110)$ & 0.140 \\
\hline PTEF $(\mathrm{mL} / \mathrm{sec}) *$ & $644(525 / 981)$ & $1257(1077 / 1541)$ & 0.008 \\
\hline PTIF (mL/sec) & $514(445 / 842)$ & $709(412 / 845)$ & 0.757 \\
\hline TEF50 (mL/sec) ${ }^{*}$ & $526(441 / 655)$ & $1201(1003 / 1411)$ & $<0.001$ \\
\hline TIF 50 (mL/sec) & $421(349 / 630)$ & $525(366 / 687)$ & 0.792 \\
\hline Phase (degrees) & $9.7(-0.2 / 12.1)$ & $3.7(-6.3 / 4.2)$ & 0.194 \\
\hline \multicolumn{4}{|l|}{$\underline{\text { Ratios }}$} \\
\hline \multicolumn{4}{|l|}{ Expiratory:Inspiratory } \\
\hline TimeExp:TimeInsp & $1.10(0.94 / 1.14)$ & $1.43(1.01 / 1.81)$ & 0.112 \\
\hline PTEF:PTIF * & $1.26(1.13 / 1.35)$ & $1.82(1.46 / 2.65)$ & 0.004 \\
\hline TEF50:TIF50 * & $1.28(1.03 / 1.36)$ & $2.03(1.91 / 2.81)$ & $<0.001$ \\
\hline
\end{tabular}

GRMD, golden retriever muscular dystrophy; RR, respiratory rate; TimeExp, expiratory time; TimeInsp, inspiratory time; TV, tidal volume; MV, minute volume; PTEF, peak tidal expiratory flow; PTIF, peak tidal inspiratory flow; TEF50, tidal expiratory flow at 50\% of TV; TIF50, tidal inspiratory flow at $50 \%$ of TV;

*

* statistically significant difference between groups. 